\title{
PORTRAIT D'UNE CLASSE MOYENNE: LES IMMEUBLES À LOYER MODÉRÉ ET LE CONFORT MODERNE À PARIS (1923-1933)
}

La mise en cuvre chaotique et incertaine du logement dit social en France, anecdotique et marginale dans un $\mathrm{XIX}^{\mathrm{e}}$ siècle tourné vers l'industrialisation, embrasse cependant l'ensemble du social en portant au débat les valeurs fondamentales et fondatrices de la société française d'alors, valeurs économiques aussi bien que sociales et morales. Les travaux pionniers de Roger-Henri Guerrand ${ }^{\prime}$ ont retracé le chemin parcouru depuis des origines balbutiantes et polémiques dans un contexte social mêlant, du côté des élites, la franche hostilité (ingérence dans la liberté d'entreprise des propriétaires), la peur (face au peuple, à ses explosions politiques et au danger de ses carences sanitaires) et le devoir de charité chrétienne.

On peut aussi voir à travers cette institutionnalisation du logement social l'histoire de la diffusion des préceptes hygiénistes, cheval de Troie des élites du $\mathrm{XIX}^{\mathrm{e}}$, associant progrès scientifique et morale bourgeoise. On peut y repérer l'évolution articulée des conceptions médicales et morales, leur montée en puissance et la place qu'elles vont prendre dans les notions de Patrie et de Nation au cours de la Troisième République, au tournant du siècle et dans l'entre-deuxguerres.

\section{Entre la Ville de Paris et l'État un aller-retour incessant: revendication, législation, programmation, modélisation}

On peut observer comment les poussées municipales parisiennes qui réclament finances et garanties d'emprunt anticipent sur la législation qui entérinera ensuite au niveau national les configurations parisiennes. L'analyse de ces processus de

\footnotetext{
${ }^{1}$ Roger-Henri GUERRAND, Les origines du logement social en France, Paris 1969. Voir aussi Susanna MAGRI et Christian TOPALOV, Reconstruire: l'habitat populaire au lendemain de la première guerre mondiale. Étude comparative France, Grande-Bretagne, Italie, États-Unis, dans: Archives européennes de sociologie 29, 2 (1988) p. 319-370; Jean-Paul FLAMAND, Loger le peuple, Paris 1989; Marie-Jeanne DUMONT, Le logement social à Paris, 1850-1930, Liège 1991.
} 
négociations permet de voir à l'œuvre l'influence des dynamiques parisiennes sur le modèle national, bien que les habitations à bon marché ne sont pas l'apanage de Paris.

Parallèlement aux premières expériences de construction d'habitations liées au contexte des grandes usines, le problème du logement des plus pauvres se pose partout dans les grandes villes de l'Europe industrielle du XIX ${ }^{\mathrm{e}}$ siècle. Des expériences commencent ${ }^{2}$, constructions $\mathrm{d}^{\prime}$ 'habitations ouvrières en particulier sous forme de coopérative. A partir de 1867, les expositions universelles internationales présentent les travaux des pays concernés en la matière et véhiculent des réflexions sur l'hygiène urbaine. La fin des années 1880 voit la création de la Société française des HBM, qui organise un premier concours pour l'étude de logements individuels et collectifs ${ }^{3}$, et voit surtout en 1894 l'adoption de la loi Siegfried ${ }^{4}$ première loi française sur les HBM. La loi Strauss de 1906 renforce les dispositions de la Loi Siegfried en réaménageant les avantages consentis et les encouragements, avec cependant deux nouveautés aux conséquences importantes: l'intervention désormais possible des communes ou départements qui peuvent consentir ou garantir des prêts aux sociétés d'HBM, leur vendre des terrains ou des constructions à prix réduit; le contrôle de la salubrité des habitations à construire, qui fait entrer une condition qualitative.

Dès les premières mesures sur l'insalubrité des logements qui suivent les années d'enquêtes et de réflexion après l'épidémie parisienne de choléra de $1832^{5}$, les efforts des commissions consultatives d'hygiène à Paris, conséquences de la Loi de $1850^{6}$, rendent perceptible l'attention et la volonté de la Ville sur le sujet. Au Conseil municipal de Paris ${ }^{7}$, les discussions aboutissent en 1884 à deux décisions qui donnent la mesure des préoccupations précoces et dynamiques de la Ville en la matière: concéder par bail emphytéotique à des particuliers des terrains appartenant à la Ville ou à l'Assistance Publique ${ }^{8}$, pour la construction

${ }^{2}$ A Lyon par exemple avec les opérations de Félix Mangini. Voir Pierre CAYEZ, Les petits logements dans les grandes villes: Lyon 1886-1968, dans: Le Mouvement social 137 (1986) p. 28-54. Pour les HBM, la création de l'office municipal parisien est pratiquement contemporaine de celles d'autres offices en province, voir Robert CHAGNY, HBM à Grenoble, 1919-1939, dans: Ibid., p. 77-108.

${ }^{3}$ Sur un terrain situé au nord de Paris, à St Denis.

${ }^{4}$ Essentiellement: création d'organes d'informations et de propagande, facilités de crédit aux sociétés qui construisent des HBM, exonérations fiscales pour les bâtiments et pour les sociétés.

${ }^{5} 1892$ verra la dernière grande épidémie de choléra à Paris.

${ }^{6}$ Sur les applications de la Loi de 1850 sur les logements insalubres, Florence BOURILLON, A propos de la Loi d'avril 1850 , la seconde République et la ville, dans: Journée d'histoire urbaine 5 mars 1997, Histoire et urbanisme, la ville et les décideurs, de la fin de l'Ancien Régime au début du XX ${ }^{\mathrm{e}}$ siècle, Université Paris XII-Val de Marne.

${ }^{7}$ Institution instable jusqu'en 1896, renouvelée tous les trois ans et privée du moyen de contrôler l'exécution de ses décisions.

${ }^{8}$ Le Conseil Municipal formulera des vœux répétés à partir de 1896 pour inciter l'Assistance 
d'Habitations à Bon Marché (HBM); mise à l'étude de quatre maisons-types par les architectes de la Ville (qui seront présentées au Conseil Municipal en 1885, mais jamais discutées).

Les créations du Musée social ${ }^{9}$ et surtout du Casier Sanitaire ${ }^{10}$ de Paris pratiquement contemporaines de l'adoption de la loi Siegfried témoignent de la recherche d'outils dans une conception innovante et qui expérimente. C'est le début de l'ère des fondations philanthropiques parisiennes qui culminera avec le retentissant concours lancé en janvier 1905 et jugé en juillet de la même année par la fondation Rothschild ${ }^{11}$ qui s'appuie sur le règlement sanitaire de Paris, institué en 1904, en application de la Loi sur la Santé Publique de $1902^{12}$, fixant des normes de surface des pièces, d'éclairement, de vue, de raccordement et de mise à disposition de l'eau potable ${ }^{13}$.

Bientôt, sous les offensives répétées des élus socialistes au Conseil Municipal, la Ville de Paris demande à pouvoir construire elle-même des HBM. Le 13 juillet 1912, par anticipation de la loi Bonnevay ${ }^{14}$ votée en décembre 1912 pour régler

Publique, qui selon le Conseil Municipal dispose d'un domaine immobilier aussi vaste qu'inexploité, à réaliser des HBM sur ses terrains. Réticente, celle-ci ne cédera qu'en 1906, avec l'opération réalisée square Delambre $14^{\mathrm{e}}$ arrondissement de Paris.

${ }^{9}$ Initiative qui rassemble des philanthropes et des réformateurs sociaux. Voir les travaux de Janet HORNE Republican Social Reform in France: The Case of the Musée Social, 1894-1914, Ph.D thesis, New York University 1992; et aussi L'économie sociale et la naissance du Musée social, dans: André GUESLIN, Pierre GULLAUME (s.l.d.d.), De la charité médiévale à la sécurité sociale. Economie de la protection sociale du Moyen Age à l'époque contemporaine, Paris 1992, p. $107-$ 116.

${ }^{10}$ Celui-ci associe dans un même fichier des informations sur un bâtiment et des données médicales sur les personnes qui y logent. Voir Yankel FIJALKOW, Mesurer l'hygiène, thèse de doctorat de géographie urbaine, EHESS 1992; ainsi que La construction des îlots insalubres, Paris 1850-1945, Paris 1998.

${ }^{11}$ DUMONT, Le logement social à Paris 1991.

${ }^{12}$ Cette loi fait obligation aux maires de toutes les communes d'élaborer selon des modèles préétablis un règlement sanitaire concernant la prévention des épidémies et la salubrité des bâtiments d'habitation. Dans les villes de plus de 20000 habitants les constructions sont soumises à permis de construire délivré par le maire à condition de satisfaire au règlement sanitaire de la commune. Mise en place d'une Administration sanitaire en charge de contrôler l'application de la loi en prononçant au besoin en cas d'insalubrité l'interdiction d'habiter, la contrainte au propriétaire d'assainir, l'expropriation et même la démolition.

${ }^{13}$ Pièces habitables: minimum $9 \mathrm{~m}^{2}$, sous combles $8 \mathrm{~m}^{2}$, avec un conduit de fumée chacune; WC intérieur et eau potable dans tout logement à partir de 3 pièces, poste d'eau à l'étage pour les autres cas.

${ }^{14}$ Véritable illustration de cet aller-retour fondateur Ville de Paris/État, cette loi est instituée en deux temps. En juillet 1912, elle consacre l'intervention municipale directe dans la construction de logements en réglant les modalités d'un emprunt par la Ville pour la construction, l'acquisition ou l'assainissement d'HBM. En décembre 1912, la loi entérinera au niveau national ces dispositifs anticipés au niveau municipal en réglant les modalités d'interventions des communes et de l'État, en introduisant une typologie officielle des logements et une relation entre montant du loyer et nombre de pièces, en créant des Offices publics d'HBM dont la compétence s'étendra de la construction à la gestion des HBM et de leurs équipements communs (lavoirs, bains, 
les modalités d'intervention entre les communes et l'État, la Ville est autorisée à contracter un emprunt pour la construction d'HBM: un arrêté préfectoral signe immédiatement l'ouverture de deux concours publics pour la conception et la réalisation d'HBM. Simultanément les HBM entrent dans les projets d'extension de la Ville puisqu'au même moment sont prises les premières décisions sur l'aménagement des fortifications déclassées. 1914 verra la création de l'Office public d'HBM de la Ville de Paris, et 1915 celle de l'Office public d'HBM du département de la Seine. Les avancées parisiennes sont un moteur pour le département et pour le niveau national qui concerne l'ensemble des autres villes.

Cela va se rejouer dans l'entre-deux-guerres: c'est le moment où les expériences parisiennes de la période précédente prennent de l'ampleur, passant de l'initiative philanthropique à la production publique municipale. Il ne s'agit plus seulement d'expérimenter des programmes et des formes architecturales au coup par coup, mais de tenter, grâce aux opportunités des terrains libérés et aux efforts de financement consentis, d'engager une politique suivie et quantitative de production d'habitations à bon marché à partir des savoirs capitalisés ${ }^{15}$ précédemment et en sollicitant le partenariat de l'État. L'aboutissement des négociations pour la démolition des fortifications à Paris, qui libèrent une emprise de terrain considérable, donne au municipalisme un lieu d'expression, et l'effet modélisateur de Paris en direction du niveau national va s'en trouver renforcé.

Il s'agit là encore des avancées de l'action municipale ${ }^{16}$ qui rencontre la question urbaine au détour des problèmes d'extension de la ville ${ }^{17}$, de gestion, ce qui aura pour conséquences de provoquer un changement d'échelle dans la vision des règlements en cours d'élaboration, et des opérations à réaliser ${ }^{18}$ et de stimuler sans relâche le législateur.

garderies, jardins ouvriers...), les villes n'ayant pas autorité pour gérer elles-mêmes leur parc d'HBM (même si elles l'ont construit).

${ }^{15}$ Cette capacité, à concevoir et à construire ces programmes particuliers, s'est expérimentée plutôt dans les sphères de la philanthropie et des réformateurs sociaux qu'au sein de l'école des Beaux-Arts, institution de formation des architectes. Voir Monique ELEB avec Anne DEBARRE, L'invention de l'habitation moderne, Paris 1880-1914, Paris 1995.

${ }^{16}$ Alain COTTEREAU, L'apparition de l'urbanisme comme action collective: l'agglomération parisienne au début, dans: Sociologie du Travail 12, 4 (1969) p. 342-365.

${ }_{17}$ Marie CHARVET, La question des fortifications dans les années 1900: esthètes, sportifs, réformateurs sociaux, élus locaux, dans: Genèses 16 (1994) p. $23-44$.

${ }^{18}$ Jean-Louis COHEN et André LORTIE, Des fortifs au périf, Paris les seuils de la ville, Paris 1992. 


\section{Associer Habitations à Bon Marché et Immeubles à Loyer Modéré pour répondre à la crise endémique du logement parisien}

Avant 1914 , le logement bénéficie d'une totale liberté en matière de loyers, l'urbanisation du XIX ${ }^{\mathfrak{e}}$ siècle notamment joue en faveur des propriétaires. De 1810 à 1910 , le prix des loyers a doublé, de façon bien plus supérieure au coût de la vie. A Paris, les recensements de 1896 et 1911 révèlent des taux de surpeuplement ${ }^{19}$ important dans les quartiers populaires. Le loyer, même s'il représente pour une famille une dépense inférieure à la part de l'alimentation, reste lourd.

Pour cause de guerre, les premiers délais moratoires sont imposés le 14 août 1914, ajournant pour les combattants, l'obligation de payer un loyer jusqu'à la fin des hostilités, en reconduisant obligatoirement et sans modification les baux venant à expiration. Il s'agit, dans l'idée qui prévaut d'une guerre courte, de protéger les familles des mobilisés. Cette mesure de guerre est donc considérée comme mesure d'exception. Pourtant le conflit s'éternise et ces mesures seront progressivement étendues jusqu'à la Loi du 9 mars 1918 qui concerne tous les Français détenteurs d'un bail ou d'une location verbale antérieure au ler août 1914, prolongeant la jouissance des lieux aux conditions du bail en cours. Le maintien dans les lieux des uns a pour conséquence la pénurie pour les autres, bien que la population ait diminué d'environ deux millions, état de fait qui trouble l'opinion publique ${ }^{20}$. En conséquence, la construction neuve privée stagne ${ }^{21}$, du

${ }^{19}$ Anita HIRSH, Le logement, dans: Alfred SAUVY, Histoire économique de la France entre les deux guerres, Paris 1984, vol. 2, p. 262-294. Elle note p. 292 sur le niveau de surpeuplement (nombre de personnes/nombres de pièces du logement) qu'à Paris, entre 1911 et 1926 le nombre de ménages de plus de 2 personnes occupant une pièce a augmenté.

${ }^{20}$ Susanna MAGRI, Consensus ou résistance populaire au réformisme social dans le domaine du logement? l'exemple du mouvement des locataires parisiens, dans: Cahiers de la Recherche architecturale, Architectures et politiques sociales 1900-1940, 15-17 (1985) p. 18-23.

${ }^{21}$ HIRSH, Le logement 1984, p. 288. Elle se livre à une série de calculs pour construire une évaluation du volume de la construction entre 1919 et 1939 , essentiellement à partir du recensement de 1962 qui pour la première fois donne l'âge des immeubles recensés, de l'enregistrement des demandes en autorisation de construire mois par mois - initiative Jean Dessirier - d'un article d'Etudes et conjonctures de juillet 1947, et d'un autre article de Lucien Flaus sur les destructions pendant la guerre de 1939-45, en septembre-octobre 1947, Journal de la Société de statistiques de Paris. Le résultat est un ordre de grandeur de 2400800 logements achevés dans la période 1915-1930, ce qui donne en moyenne 96000 logements par an et 114000 logements pour les seules années 1919-1939, tenant pour nulles les constructions de 1915 à 1918. Ces estimations fragiles donnent dans l'hypothèse la plus favorable un rythme de construction égalant l'avant guerre pour une population plus nombreuse. Pour la construction subventionnée elle retient le chiffre de 300000 logements de 1919 à 1939 avancé par Lucien Flaus, La construction d'immeubles d'habitation en France de 1928 à 1939, mars-avril 1946, Journal de la Société de statistiques de Paris. $75 \%$ de ces 300000 logements ayant été achevés après 1928 . Pendant la période 1930-33,40\% des constructions neuves auraient été financées à l'aide du crédit public. 
fait du régime des taxations défavorables aux propriétaires. Pour suivre le coût de la construction les loyers auraient dû être multipliés par 9 ou 10. Mais la crise économique générale est une des causes de ce volume réduit de construction. Le propriétaire rentier vivant uniquement du revenu de ses immeubles doit trouver d'autres moyens de vivre, ou accepter un niveau de vie réduit par rapport à 1914, (de moitié en 1935, du tiers en 1939), et n'assurer ni entretiens ni réparations. Le blocage de cette dynamique a supprimé pour ainsi dire le marché et c'est l'autofinancement en copropriété (innovation) ou lotissements de banlieue ${ }^{22}$, voire même l'impulsion de l'autoconstruction par les ménages ${ }^{23}$, le plus souvent aussi en banlieue et les initiatives publiques de construction de logements aidés qui vont permettre à la construction de garder un rythme de croisière.

Dans le contenu de ces initiatives publiques réside une véritable originalité parisienne dans les options retenues pour agir sur la crise du logement: en effet une partie des élus, se saisissant d'un des articles de la loi du 19 avril 1919 sur le déclassement de l'enceinte fortifiée, va parvenir à convaincre l'ensemble du Conseil municipal de la nécessité opportune d'élargir à la classe moyenne le bénéfice des logements municipaux que la Ville a le projet de construire. En effet, cette loi sur le déclassement de l'enceinte établit pour la réaffectation des terrains une servitude de non aedificandi sur la zone, qui doit être aménagée en espaces libres à l'exception d'édifices publics, et d'une part de terrains affectés à la construction d'Habitations à Bon Marché et d'Habitations à Loyer Modéré ${ }^{24}$ "destinées aux familles nombreuses de la classe moyenne". On retrouve en 1922 cette notion d'"habitations à loyer modéré" (qui n'avait cependant pas été définie techniquement et juridiquement en 1919) qui prend de l'importance dans les débats du Conseil Municipal concernant les programmes d'édification d'Habitations à Bon Marchée en $^{25}$ dans lesquelles les programmes d'Habitations à Loyer Modéré se trouvent encore inclus. Le principal conflit porte sur les bénéficiaires prévus de ces HLM ou ILM (immeuble à loyer modéré). Quelle est cette classe moyenne évoquée? S'agit-il des adhérents de la Confédération des Travailleurs Intellectuels (fondée en 1920) qui compte dans ses rangs professions libérales, universitaires, artistes, ou bien s'agit-il de la masse plus anonyme des petits employés, modestes fonctionnaires et autres petits salariés? Le débat oppose d'une part les tenants de l'ouverture de la "sollicitude publique" en direction de cette classe moyenne "armature de notre démocratie" prise dans son ensemble ou

\footnotetext{
${ }^{22}$ Annie FoURCAULT, Les lotissements défectueux en région parisienne: un exemple de gestion technique d'une crise urbaine, dans: Yves COHEN, Rémi BAUDOUï (dir.), Les chantiers de la paix sociale, 1900-1940, Fontenay St Cloud 1996, p. 255-264.

${ }^{23}$ Susana NUNEZ, Le concept de logement mis en place dans la région parisienne, 1910-1950, Paris EHESS, Thèse de doctorat en cours.

${ }^{24}$ Terme qui résulte d'une improvisation d'un député lors de la séance de discussion, un contenu plus précis lui sera donné en 1923.

${ }^{25}$ Procès-verbaux des débats du Conseil municipal de Paris: séance du 13 juillet et du 28 décembre 1922.
} 
sa diversité, qui ne doit pas être laissée pour compte car elle souffre autant que les autres classes des bouleversements économiques de l'après-guerre et en particulier la multiplication par 3,5 du montant des loyers, et d'autre part ceux qui estiment que rien ne peut ni ne doit être détourner de l'aide à accorder aux plus pauvres. Pour les premiers, il s'agit de montrer que les opérations d'HBM constituent des opérations financières coûteuses et déficitaires par essence alors que les opérations d'ILM se paieront toutes seules par le niveau de revenus plus élevés des locataires envisagés, leur solvabilité plus stable et l'excédent de recettes fiscales provenant des taxes mobilières afférentes aux appartements. Pour les seconds, l'argumentaire repose sur un septicisme général devant la qualité des locataires espérés, le sentiment de gaspillage face aux besoins criants des plus démunis. Ce septicisme, bien qu'il conduise de longues discussions, ne fera pas le poids devant un Conseil municipal où la classe moyenne version "travailleurs intellectuels" semble bien représentée.

En effet, près plus d'une année d'études et de débats plus tard, ces débats aboutissent en juin 1923 à l'organisation de quatre concours d'architecture pour des "immeubles à loyer modéré" à édifier sur l'emplacement des anciennes fortifications, et à la désignation des architectes lauréats en juillet de la même année. La négociation pour la définition de la gestion des opérations débouche sur une convention passée avec un consortium bancaire dont est issue la Régie Immobilière de la Ville de Paris (RIVP).

Là encore les délibérations et les programmes de la Ville anticipent sur les lois Loucheur (1928) et Bonnevay (1930) instituant une politique d'intervention financière directe de l'État au niveau national. En 1928, La Loi Loucheur prévoit pour la première fois simultanément des programmes de construction d'envergure (260 000 logements à construire entre 1928 et 1933, dont 60000 logements de formule "Immeuble à Loyer Modéré") et des mesures financières destinées à leur réalisation (avances et prêts à taux réduits pour la construction mais aussi encouragement à l'accession à la petite propriété). A Paris, une des conséquences principales $^{26}$ sera la création de la Société Anonyme de Gestion Immobilière (SAGI), issue d'une convention entre la Ville et une société immobilière, qui va prendre en charge le relogement des expropriés des opérations de voirie dont le coût serait équilibré par des logements "de luxe" (d'où le contraste des opérations) hors du cadre de la Loi Loucheur. En 1930, La Loi Bonnevay prévoit la création des HBM améliorés (HBM A), type intermédiaire entre l'HBM et l'ILM (ou HLM). Logements destinés aux petites classes moyennes, leurs surfaces sont dilatées par rapport aux HBM "ordinaires" et leurs équipements sont moindres par rapport aux ILM ${ }^{27}$. L'année 1934 verra l'arrêt du programme Loucheur.

\footnotetext{
${ }^{26}$ DUMONT, Le logement social à Paris 1991, p. 141. Les sociétés SGIM et CPEG furent créées pour faire contre poids dans l'opinion face aux "privilèges" des opérations confiées à la SAGI, elles réalisèrent 3468 logements.

${ }^{27}$ Ibid., p. 167. Tableau récapitulatif qui montre un gonflement de plus de $10 \mathrm{~m}^{2}$ en moyenne pour
} 


\section{Le confort moderne, mythes et réalités}

Le rôle des hygiénistes ${ }^{28}$ dans la question urbaine a été souligné, mais ce rôle ne s'arrête pas aux questions d'aménagement urbain. En effet, ils revendiquent l'exercice de compétences et d'expertise dans l'aménagement même des logements. Lire l'ouvrage rédigé par le professeur Courmont ${ }^{29}$, un médecin lyonnais, montre par exemple comment se construit leur argumentaire qui fait de l'hygiène le point d'appui d'une population saine, forte et fournie. L'hygiène n'est pas palliative mais éminemment préventive, à ce titre elle doit intervenir dans toute action et bien au delà du domaine de la santé: c'est à cette condition que la France retrouvera son prestige. Le texte en passe d'ailleurs par des injonctions pour la construction de logements sains face aux "maisons meurtrières". Outre une exigence pour des matériaux et un environnement salubre, les descriptions des intérieurs stigmatisent les recoins et scandent la notion de nettoyage: tout doit être lavable, les tapis sont criminels à cause des poussières - peut-être malsaines - qui s'y logent, ainsi que le recours à des équipements techniques sanitaires généralisés.

Or, même si les nouvelles possibilités induites par l'évolution rapide de l'innovation technique ont une existence réelle, leur diffusion plus large suit un rythme bien lent dans l'équipement des habitations: absence à la fois dans les taudis et dans les résidences patrimoniales des classes supérieures, où une domesticité toujours très présente exécute entre autres les différentes "vidanges"; timides avancées par éléments isolés dans les appartements en location, par les locataires eux-mêmes (installation de l'électricité dans une pièce seulement, aménagement d'un cabinet de toilette, achat d'un chauffe-eau portatif... $)^{30}$. L'ensemble de ces dispositifs d'hygiène mais aussi d'amélioration technique des habitations va être rassemblé sous le nom de confort moderne. Et au delà d'une simple qualification technique matérielle, cette appellation de confort moderne va devenir un label qualifiant un mode de vie.

En effet, le Conseil municipal de Paris, dans la série de débats ${ }^{31}$ de 1922 sur les habitations à construire par la Ville, va articuler immeubles à loyer modéré, classes moyennes et confort moderne. Il va faire de cette notion de confort moderne le pivot de l'élaboration des opérations d'ILM. Que faut-il entendre ici par confort moderne? Il s'agit à la fois d'équipements techniques et de dispositifs

les HBM A par rapport aux HBM, alors que les surfaces HBM A-ILM sont sensiblement les mêmes.

${ }^{28}$ Lion MURARD, Patrick ZYLBERMAN, L'hygiène et la République, Paris 1996.

${ }^{29}$ Précis d'hygiène, Paris 1914.

${ }^{30}$ Mémoire de Paris 1919-1939, Bibliothèque Historique de la Ville de Paris, enquête de 1993.

${ }^{31}$ Conseil municipal de Paris. Rapports et documents $N^{\circ} 30,53$ et 71 (1922). Mémoires préfectoraux $\mathrm{N}^{\circ} 2$ et $12(1922)$. 
architecturaux: "chauffage central, ascenseur, salle de bains, escalier de service". En plus peut-être une "vaste antichambre, si nécessaire à tous ceux qui sont professionnellement $t^{32}$ condamnés à recevoir nombre de visiteurs". Et pour contrer les protestations évoquant le luxe de l'escalier de service par exemple, les arguments vont portés sur ce confort comme une nécessité professionnelle des travailleurs intellectuels, "le noble peuple de Paris est trop compréhensif et trop fin pour ne pas comprendre par exemple que le salon, qui est pour certains travailleurs un luxe inutile, constitue pour d'autres (médecins et avocats par exemple) l'indispensable complément du logis familial'. Autre argument, de nature financière cette fois, la possibilité d'augmenter la valeur locative des appartements par le niveau de prestations fournis par le confort moderne, ce qui rend leur installation rentable. Dans le même ordre d'idée la présence du confort moderne permettra aux ILM de rester, dans le futur, concurrentiel avec la construction privée qui, elle aussi, le proposera dans ses programmes quand elle redémarrera.

Le confort moderne est finalement ici compris plus comme une affaire de classe que de revenu, plutôt comme une affaire de mode de vie que d'ostentation. Et ainsi on va assister dans la définition des programmes d'opération d'ILM à la scission en deux types, confort moderne et confort réduit (sans ascenseur, sans escalier de service, sans salle de bains ni antichambre ... mais ne reprenant pas "pour autant la formule étriquée des HBM"), dont les loyers varieront aussi en fonction de la surface, reprenant l'effort initial demandé en faveur des familles nombreuses dans la loi de 1919.

L'hypothèse que formulent les édiles, en associant une identité de groupe (de classe) à une configuration d'habitat, postule l'efficience d'une culture citadine porté par les formes d'innovation des habitations collectives, fruits d'une politique sociale en plein essor. Postulant pour les plans des logements des Immeubles à Loyer Modéré parisiens un destin de modèle plus large que ce que l'on pourrait croire au premier abord, ces premiers programmes sociaux à destination de la classe moyenne me paraissent représenter une réflexion sur les différenciations sociales entre les groupes des habitants destinataires. Ces plans, pris ici comme une des figures de discours, permettent de suivre les représentations que se font les édiles et le cercle d'experts et de professionnels qu'ils mobilisent, de tel ou tel groupe social à partir des dispositifs et des équipements qu'ils vont leur fournir.

${ }^{32}$ C'est l'auteur du rapport qui souligne. 


\section{Un premier bilan}

Aux environs de 1910 soit une quinzaine d'années après la Loi de 1894 sur les HBM, le bilan des constructions issues des fondations philanthropiques, de particuliers et de 80 sociétés approuvées, est de 9780 logements (314 immeubles et 226 maisons individuelles). L'intervention financière de l'État sous toutes ses formes représentent environ 8 millions alors que les seules fondations Lebaudy et Rothschild représentent à elles deux la mise en ouvre de 20 millions.

En ce qui concerne les vingt années de l'entre-deux-guerres, au total la Ville de Paris a réalisé 32751 logements de type HBM, HBM A et SAGI 1 et 11221 logements ILM + 10391 SAGI 2, soit 21610 logements pour les classes moyennes. A cela il faut ajouter environ 15700 logements HBM construits par l'office du département de la Seine et quelques milliers supplémentaires réalisés par les offices municipaux de banlieue.

Ce résultat qui totalise la réalisation d'environ 50000 Habitations à Bon Marché et 20000 logements en Immeuble à Loyer Modéré pour la Seine, laisse l'agglomération parisienne loin derrière Berlin (120 000 logements) et Londres (250 000 logements) pour la même période.

Les différentes logiques qui s'affrontent autour de ces questions de logement mettent en relief des formes de représentations et des aspects de l'univers mental de configurations d'acteurs du monde urbain entre $\mathrm{XIX}^{\mathfrak{e}}$ et $\mathrm{XX}^{\mathfrak{e}}$ siècle, mobilisés et activés dans leurs enjeux de pouvoir. Les critères d'usages de l'espace qui permettent de dessiner les contours d'un mode de vie et qui l'associent par des pratiques supposées à une forme d'identité sociale, permettent de percevoir cet espace du logement utilisé pour jouer un rôle dans la formation de l'identité de la classe moyenne.

Les revendications multipliées et les recherches persévérantes des hygiénistes autour des logements économiques, et cela bien avant l'entre-deux-guerres dans les fondations philanthropiques notamment, semblent camper ces hygiénistes dans un rôle moteur dans l'évolution des logements en particulier dans la notion d'équipement et leur systématisation.

Cette histoire longue de plus d'un siècle, dont seule une étape a été évoquée ici, avec ses évolutions juridiques aussi bien qu'économiques, permet d'apercevoir le cheminement qui va des initiatives privées vers l'élaboration de structures institutionnalisées. Le caractère le plus progressiste peut-être de cette période est l'avancée législative dont témoignent ces réalisations de logements sociaux français, qui constitue tout de même une première brèche dans le principe sacrosaint de la propriété. 


\section{Deutsche Zusammenfassung}

Der Beitrag postuliert für die Sozialwohnungen der "Immeubles à Loyer Modéré", wie sie in der Zwischenkriegszeit in Paris von den kommunalen Instanzen gemäß den neuen, erst seit 1914 bestehenden Zuständigkeiten gebaut wurden, einen weitaus stärkeren Modellcharakter, als man zunächst annehmen würde. Es soll gezeigt werden, wie sich in diesen ersten Sozialprogrammen für die Mittelklasse die Vorstellungen finden lassen, welche die Stadtväter von der Zielgruppe ihrer Bemühungen hatten. Diese Vorstellungen, die aus einem Gemenge von öffentlichen Debatten, wirtschaftlichen, gesetzlichen und urbanen Zwängen, aber auch aus Plänen und früher realisierten Experimenten erwachsen, sind unter anderem dadurch charakterisiert, daß sie die Bewohner in verschiedene Gruppen mit einem identifizierbaren und homogenen wirtschaftlichen und sozialen Verhalten teilen. Für jede dieser Gruppen haben die Stadtväter ein Register normierter Praktiken für das häusliche Leben. Diese normativen Vorgaben finden dann ihre materielle Ausprägung in den räumlichen Vorrichtungen der geplanten Wohnungen. Dadurch wird die Mittelklasse gleichbedeutend mit "Immeubles à Loyer Modéré". Als sie einen größeren räumlichen und sozialen Umfang annehmen, deuten diese öffentlich geförderten Wohnungen durch die Bereitstellung "moderner" Ausstattung einerseits auf eine Art Internalisierung der Hygieneregeln und -praktiken, andererseits auf eine Aufwertung eines "modernen" Lebensstils hin. Schaut man sich die Diskussionen um das vorgeschlagene Niveau sanitärer und technischer Wohnungsausstattung näher an, um den Begriff "confort moderne" zu verstehen, so läßt die Deutung dieser Einrichtungen erkennen, auf welche Art eine räumliche Anlage zur Unterstützung des Verhaltens einer sozialen Gruppe dienen kann. Womit sich auch die Hypothese prüfen läßt, wonach der Platz der Wohnung durch seine räumliche Konfiguration an der Konstruktion sozialer Identităten beteiligt ist. Das Lesen der Pläne dieser Wohnungen als einer der Figuren im Diskurs um den Lebensstil erlaubt es dann, die identitätstiftenden vornehmen häuslichen Verhaltensweisen zu rekonstruieren. Die Stadt als Herd der Innovation und als Ort der Verbreitung dieser Modelle erscheint hier auf Umwegen als Anführerin im Handeln angesichts der sozialen Frage. Mit Hartnäckigkeit drängt sie einen ängstlichen Staat, der sich bald diese kommunalen Experimente zueigen macht, zu einer umfassenderen Wohnungspolitik.

\section{English Abstract}

This paper argues that housing of Parisian administrations by "Immeubles à Loyer Modéré" during the inter-war-period based on prerogatives only from 1914 on, had a more far-reaching model character than one would expect on the first look. It is shown how the images can be found in these first social programs destined for the middle class, that the city fathers had in mind of the target group of their actions. These ideas, taking their rise from discussions, economic, legislative and urban constraints, but also from projects and earlier experimental realisations, are characterised by considering the inhabitants being divided into differents groups with identifiable and homogeneous economic and social behaviour. The city fathers make up for every group a register of standardized practices concerning the domestic life. These normative registers find their materialisation in the spatial organisation of the planned apartments. Thus the middle class becomes equivalent to the "Immeubles à Loyer Modéré". Assuming a larger spatial and social extent this housing signalizes by making available "modern" equipments, a sort of internalisation of hygienic rules and practices and an upgrading of a "modern" life-style. Detailing the debates on the level proposed for sanitary and technical equipment of the apartments in order to see the meaning of the "confort moderne", the interpretation of these facilities leads to realize the way a spatial disposition can be used for supporting a practice attributed to a social group. By doing this the hypothesis can be tested that the space of housing according to its spatial configuration participates in shaping social identities. Interpreting the designs of these housings as a figure of 
discourse on the ways of life then permits to reconstruct the distinguished domestic behaviour which causes identity. The city as a cradle of innovation and place of diffusion of these models thus appeard indirectly as instigator of action facing the social question by urging stubbornly the timid state which soon adopted the cities experiences, to a more extensive housing policy. 